parameters are returned for follow up providing further opportunities for the ACP team to engage with the practice.

Results $87 \%$ of general practice teams in Canterbury have supported patients to create an eACPlans.

General practice teams create $80 \%$ of the Canterbury's eACPlans.

Increased multidisciplinary approach to plan creation.

Conclusion(s) A cross-system approach to implementation has facilitated the establishment of ACP in general practice.

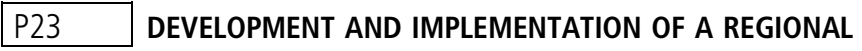 ELECTRONIC ACP PLATFORM IN THE SOUTH ISLAND OF NEW ZEALAND}

J Goodwin*, J Large, G Young. Canterbury Initiative, Christchurch, New Zealand

\subsection{6/spcare-2019-ACPICONGRESSABS.109}

Background The South Island Alliance (SIA) strives for innovative and efficient health services by bringing together the five South Island District Health Boards (DHBs) to work collaboratively. ACP is a focus area for the SIA leadership team. The Health of Older People Service Alliance has been endorsed to provide oversight and leadership to support SI DHBs develop and embed electronic Advance Care Plans (eACPlans).

Methods a four step approach

Step 1 - Systems development: The creation of a single ACP platform in the electronic medical record enabling eACPlans to be created, shared and viewed across services and systems throughout the region.

Step 2 - Quality processes: A South Island Regional quality Verification (SIRV) team has been established to implement the SIRV processes and ensure the content of SI eACPlans, are clinically interpretable and can be confidently used by clinicians to guide care and ensure patient wishes are honoured.

Step 3 - Implementation support: Development of a regional workbook, communication releases \& clinical pathway. Regular teleconference meetings are held to provide a platform for DHB ACP leads and SIRV team members to share ideas and develop 'broadly similar' approaches to support the roll out of the eACP.

Step 4 - Regional measures and reports:

Results Steps 1,2 \& 3 have been implemented and the coordinated 'go live' date for the project has been agreed by the 5 DHBs for early December 2018.

Conclusion(s) Regional implementation of eACP in the SI is helping to optimise efficiency and encourage a broadly similar approach.

\section{P24 PREDICTING LIFE EXPECTANCY TO AID IN ADVANCE CARE PLANNING}

${ }^{1} \mathrm{M}$ Beeksma*, ${ }^{2} \mathrm{~A}$ Suntjens, ${ }^{1} \mathrm{~A}$ van den Bosch, ${ }^{1} \mathrm{H}$ Das, ${ }^{2} \mathrm{G}$ Westert, ${ }^{3} \mathrm{M}$ Numans, ${ }^{1} \mathrm{H}$ Hendrickx, ${ }^{2} Y$ Engels, ${ }^{2} \mathrm{~A}$ Groenewoud. ${ }^{1}$ Radboud University, Nijmegen, Netherlands; ${ }^{2}$ Radboudumc, Nijmegen, Netherlands; ${ }^{3}$ Leiden University Medical Center, Leiden, Netherlands

\subsection{6/spcare-2019-ACPICONGRESSABS. 110}

Background Accurate timing of ACP is considered challenging. Available tools are not widely implemented, can be unfeasible to screen large populations, and require prior awareness to be used proactively in individual patients. We are developing an automated signaling tool for early identification of patients who might benefit from ACP. In this talk, we will focus primarily on the model we created to predict life expectancy, which we consider to be a first step towards identifying these patients. Additionally, we will discuss further model development and deployment in a real-world setting.

Methods We used machine learning (ML) and natural language processing (NLP) techniques to train a recurrent neural network on 1234 medical records of deceased patients. We trained several models, and compared the best-performing model to doctor's performance on a similar task as described in the literature.

Results While doctors were correct in 20\% of the cases (allowing an error margin of 33\% around the actual moment of death), our best-performing model attained a prognostic accuracy of $29 \%$. Being overly optimistic about life expectancy harms anticipation to end-of-life care. Our model was less likely to overestimate life expectancy (in 31\% of the incorrect prognoses) than doctors (63\%) were.

Conclusions Our research shows that ML and NLP offer a feasible approach to predicting life expectancy. The results are promising, given that our model is trained on a relatively small data set. Current work focuses on further model development with an increased dataset, and implementation of the tool in primary care facilities.

\section{\begin{tabular}{|l|l}
\hline P25 FREQUENCY AND CONTENTS OF ADVANCE CARE \\
\hline
\end{tabular} PLANNING DISCUSSIONS IN PRIMARY CARE}

${ }^{1} \mathrm{~J} \mathrm{Hamano}{ }^{*},{ }^{2} Y$ Kizawa. ${ }^{1}$ University of Tsukuba, Tsukuba, Japan; ${ }^{2}$ Kobe University Graduate School of Medicine, Kobe, Japan

\subsection{6/spcare-2019-ACPICONGRESSABS.111}

Background General practitioners (GPs) are well placed for identifying patients in need of advance care planning (ACP) and initiating ACP before acute deterioration in their illness trajectory. However, little is known about the frequency and contents of ACP discussions in primary care. We aimed to explore the frequency and contents of ACP discussions among patients, family members, and GPs.

Methods A multicenter, cross-sectional, observational study was conducted at 17 clinics with 22 GPs. In March 2017, each GP set an arbitrary day in advance and we enrolled all patients aged $\geq 65$ years who visited the GPs on that day. We explored the frequency and topics of ACP discussions. We identified patients at risk of deterioration and dying based on the Japanese version of the Supportive and Palliative Care Indicators Tool (SPICT ${ }^{\mathrm{TM}}$ ).

Result In total, 382 patients with a mean age of $77.4 \pm 7.9$ years were included. Twenty-three patients (6.0\%) had ACP discussions with their GP. Of 66 patients at risk of deterioration and dying, 13 patients (19.7\%) had ACP discussions with their GP. The most common topic among patients overall was anticipated declines in activities of daily living (ADLs), but the topic of surrogate decision-makers was the most common with neurological patients.

Conclusion Primary care patients aged $\geq 65$ years did not necessarily have frequent ACP discussions with their GP, but discussions were more common with patients at risk of deterioration and dying. The anticipated declines in ADLs was most frequently discussed topic among patients, family members, and GPs. 